\title{
Thickness and dielectric constant determination of thin dielectric layers
}

\author{
Helene E. de Bruijn, Marcel Minor, Rob P.H. Kooyman and Jan Greve \\ Technical Optics Group, Faculty of Applied Physics, University of Twente, P.O. Box 217, 7500 AE Enschede, The Netherlands
}

Received 24 April 1992

\begin{abstract}
We derive a method for the determination of the dielectric constant and thickness of a thin dielectric layer, deposited on top of a thick dielectric layer which is in turn present on a metal film. Reflection of $p$ - and s-polarized light from the metal layer yields minima for certain angles of incidence where the light is absorbed by the metal. The thin dielectric layer causes shifts in the angles at which the minima occur, from which the thickness and dielectric constant can be obtained. The model is tested for 3.5 and $14 \mathrm{~nm}$ thick photoresist gratings.
\end{abstract}

\section{Introduction}

We want to characterize a thin biological layer by its thickness $d$ and dielectric constant $\epsilon$, preferably in a spatially resolved way. Several methods have been described, which allow such a characterization:

Ellipsometry characterizes a thin dielectric layer on top of a substrate by measuring the change in ellipticity of a reflected light beam in combination with the reflected intensity [1].

In waveguide structures an additional dielectric layer on top of the waveguide causes a change in propagation constant for TE and TM modes from which the additional layer can be characterized [2].

A thin dielectric layer on top of a metal film creates a shift in the angle of incidence at which surface plasmon resonance (SPR) occurs. By measuring this shift for bulk media with different refractive indices the layer can be characterized [3].

The first two techniques cannot be used however to characterize a layer in a spatially resolved way, which is important for inhomogeneous layers as e.g. lipid monolayers with crystal domains. The third method can be combined with imaging in a surface plasmon microscope [4] to characterize layers spatially resolved. Application of this method on the other hand requires the use of liquid bulk media, whereas lipid monolayers often have air as a surrounding medium.
Therefore we have extended the technique of Thiefenthaler and Lukosz [2] to a method which uses the electron resonances excited by light in a metal film covered by a thick dielectric layer [5]. These resonances can be measured as minima in the reflected intensity for one or more angles of incidence, the so-called resonance angles. The number of minima observed depends on the thickness of the dielectric layer, and the resonance angles are different for s- and p-polarized light [5].

The resonance angles, at which the minima occur, shift for both p-and s-polarized light if an additional thin layer is introduced on top of the layer structure (fig. 1). These shifts in resonance angle depend for p- and s-polarization in a different way on the thickness and dielectric constant of the additional thin layer. Therefore the thickness and dielectric constant can be found separately.

By imaging the illuminated area of the metal surface with the reflected light the new technique can be applied to characterize for example single crystalline domains in a lipid layer.

Here the accuracy of the method is discussed and the results for test layers are presented. 


\section{Theory}

In order to characterize the thin dielectric layer 4 on top of the layer system: metal 2 and thick dielectric layer 3 in fig. 1, the thickness and dielectric constant of metal and thick dielectric layer have to be known. This means that the characterization is done in three steps:

\section{Metal}

The metal film is characterized from the SPR reflection curve of the bare metal [6].

\section{Thick layer}

In order to characterize the thick dielectric layer 3 the Fresnel equations are used to fit the calculated position of the reflection minimum to the measured resonance angle $\theta_{\text {res. }}$. For each resonance angle measured using one polarization, however, there exists for each arbitrarily chosen thickness a dielectric constant such that the layer structure has a reflection minimum at the measured angle. In ref. [7] we showed that for an arbitrarily chosen dielectric constant several thicknesses yield the same resonance angle. These thicknesses can be calculated for each dielectric constant with the following equation [7]:

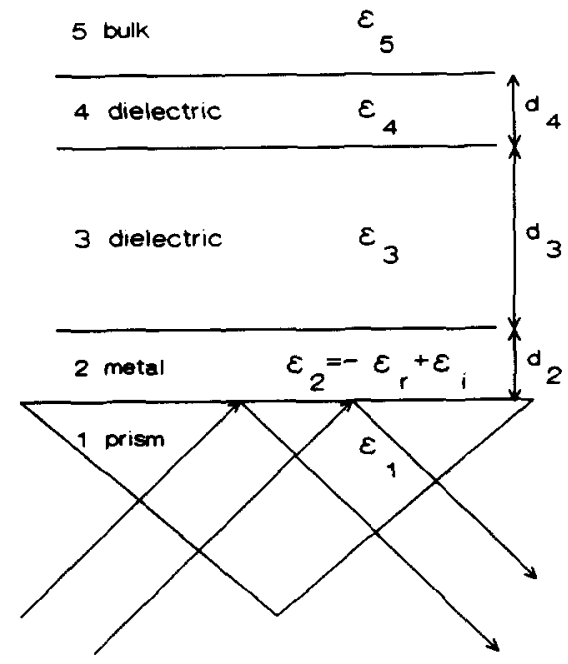

Fig. 1. The layer structure on top of the prism illuminated with a laser beam. $d_{3}=d_{3}^{0}+m d_{3}^{*}, \quad$ with $d_{3}^{*}=\frac{\lambda}{2 \sqrt{\epsilon_{3}-\epsilon_{1} \sin ^{2} \theta_{\text {res }}}}$.

In this formula $d_{3}^{0}$ is the thickness that follows from SPR [3].

From the details of the deposition of layer 3 it is usually possible to get an estimate of the proper order $m$ of the thickness as $d_{3}^{*}$ is several hundred $\mathrm{nm}$. An alternative is to obtain the proper thickness from the calculated width of the reflection curve, which also depends on the order $m$. Due to the difference in sensitivity for layer 3 of s- and p-polarized light, two intersecting lines in the $\epsilon_{3}-d_{3}$ plane are obtained (fig. 2). The intersection point yields the proper $\epsilon_{3}$, $d_{3}$ combination which describes the two respective reflection minima.

\section{Thin dielectric layer}

Now that layer 3 has been characterized the same method can be used for layer 4 . As layer 4 is thin the order $m$ of the thickness can be put at 0 .

As we are only interested in the characterization

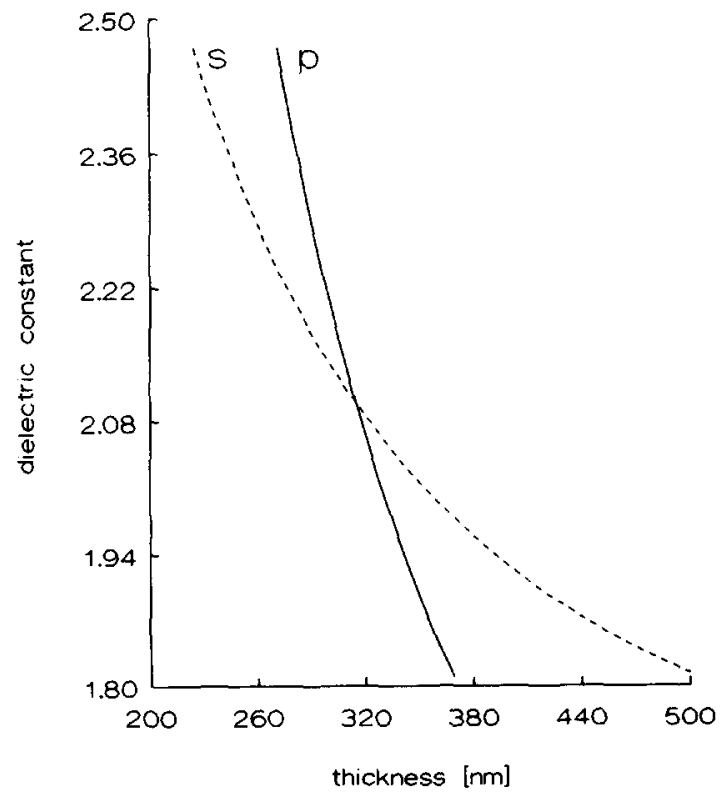

Fig. 2. The shift in resonance angle for s- and p-polarized light leads to $\epsilon_{3}, d_{3}$ combinations which form two intersecting lines in the $\epsilon_{3}-d_{3}$ plane for each order number $m$ of the thickness. 
of the thin dielectric layer the choice of layer 3 is free. In ref. [7] it is shown that the shift in resonance angle due to the additional layer 4 becomes smaller for increasing order $m$ of thickness $d_{3}$.

Further it is shown that at least order $m=1$ is necessary to obtain resonance for s-polarization. So order $m=1$ is chosen for the thickness of layer 3 $\left(d_{3} \approx 300 \mathrm{~nm}\right)$.

\section{Materials and methods}

For measuring the angle dependent reflection curves a turntable with steps of $0.01^{\circ}$ was used as in ref. [6], with a laser diode $(\lambda=675 \mathrm{~nm})$ instead of a HeNe laser. The reproducibility of determining absolute angles in this setup is $0.04^{\circ}$. We have characterized the metal film with this equipment.

For the microscopical measurements the apparatus of fig. 3 is used, which is described in more detail elsewhere [8]. It consists of a laser diode $(\lambda=675$ $\mathrm{nm}$ ) and $\mathrm{a} \lambda / 2$ plate, to change the polarization direction, mounted on an incoupling arm which can be rotated around the prism. On the outcoupling arm two microscope objectives are mounted with a camera to image the illuminated area of the metal surface. The angle of incidence is measured with a potentiometer. The shift in resonance angle can be measured with an accuracy of $0.02^{\circ}$. This setup is used to characterize the thick and thin dielectric layers.

For the metal film silver was chosen as this metal gives reflection curves with the smallest halfwidth [9]. The silver films were evaporated $(2.5 \mathrm{~nm} / \mathrm{s}$ at

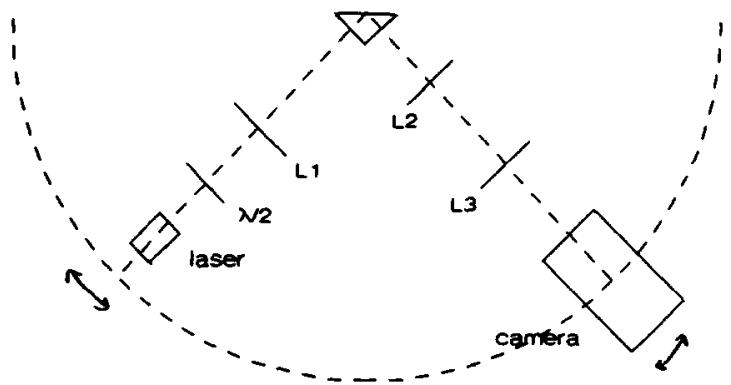

Fig. 3. The microscope setup consisting of an incoupling arm with a laserdiode and $\lambda / 2$ plate, the prism and the outcoupling arm with imaging lenses and $C C D$ camera. $\left.1 \times 10^{-6} \mathrm{mbar}\right)$. The variation in plasmon resonance angle over the evaporated area $(20 \times 20 \mathrm{~mm})$ is less than $0.04^{\circ}$, which has a neglectable influence on the subsequent characterization of the thin dielectric layer.

As we want to apply the method for studying biological samples, which are usually deposited on microscope slide glasses, $\mathrm{SiO}_{2}(\epsilon \approx 2.1)$ was chosen as material for the thick dielectric layer, which has as a benefit that it is a stable material. An additional advantage of $\mathrm{SiO}_{2}$ is that it is a hydrophilic substrate, which is necessary for the preparation of lipid monolayers. The $\mathrm{SiO}_{2}$ layers were sputtered $(0.1$ $\mathrm{nm} / \mathrm{s}$ at $1.2 \times 10^{-2} \mathrm{mbar} \mathrm{Ar}$ ). With the microscope of fig. 3 we found that the resulting $\mathrm{SiO}_{2}$ layers had a thickness gradient of $2-5 \mathrm{~nm} / \mathrm{mm}$. This leads to difference in resonance angle of about $0.1^{\circ}$ over $1 \mathrm{~mm}$, which is the illuminated area in the turntable setup. So in the turntable setup we would only measure an average reflection curve. This means that these $\mathrm{SiO}_{2}$ layers can only be studied with the microscope setup as they have to be characterized spatially resolved.

For the test layer a thin photoresist (microposit 1400 , Shipley, Coventry $\epsilon \approx 2.78$ ) layer was spincasted on top of the $\mathrm{SiO}_{2}$ and a grating pattern as in fig. 4 was made with photoresist tracks of $80 \mu \mathrm{m}$ width and bare $\mathrm{SiO}_{2}$ tracks of $30 \mu \mathrm{m}$ width.

The tracks are lined parallel to the plane of incidence in the microscope. In this way interference effects similar to the ones observed in surface plasmon resonance microscopy [10] are avoided, as these ef-

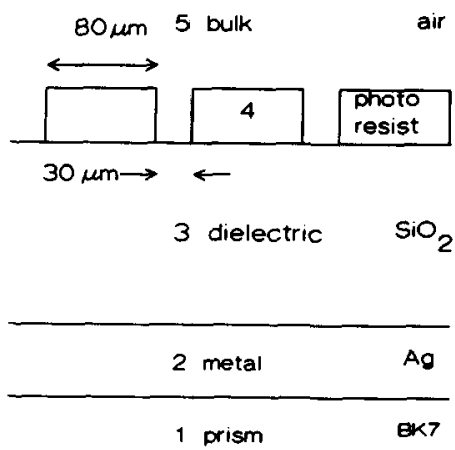

Fig. 4. Cross section of the test grating on top of the $\mathrm{SiO}_{2}$ layer and metal film. The photoresist tracks are $80 \mu \mathrm{m}$ wide and the bare $\mathrm{SiO}_{2}$ tracks are $30 \mu \mathrm{m}$ wide. 
fects occur only at boundaries between bare and covered $\mathrm{SiO}_{2}$ perpendicular to the plane of incidence.

\section{Accuracy}

In order to characterize the thin dielectric layer 4 three layers have to be characterized. The influence of the inaccuracy with which the different reflection minima are determined, on the accuracy of the characterization of the different layers, was tested by simulating the following test layer structure with the Fresnel equations for $\lambda=675 \mathrm{~nm}$ :

5: Air,$\quad \epsilon_{5}=1$,

4: Test layer $, \quad \epsilon_{4}=2, \quad d_{4}=5 \mathrm{~nm}$,

3: $\mathrm{SiO}_{2}$ layer $, \epsilon_{3}=2.1, \quad d_{3}=315 \mathrm{~nm}$,

2: Silver film $, \epsilon_{2}=-19+0.65 \mathrm{i}, d_{2}=53 \mathrm{~nm}$,

1: Prism , $\quad \epsilon_{1}=2.3$.

Each reflection curve necessary for the characterization was simulated using the exact data and shifted subsequently with the maximum inaccuracy in measuring $\theta$, which was in our case $0.04^{\circ}$ for an absolute angle and $0.02^{\circ}$ for an angle measured relative to another angle.

Just like in the experimental situation the fitting procedure as described in the theory part was applied to characterize the layer. For each following layer the characteristics, as determined using the shifted resonance angles of the already characterized layers, were used.

The influence of these angle shifts on the determined characteristics of layer 4 are given in table 1 , expressed in percentages of the exact value.
From table 1 it is clear that the inaccuracy in characterizing layer 4 is mainly determined by the accuracy with which the angle shift due to layer 4 itself can be measured. This can be understood as the relative inaccuracy in angle shift is larger for smaller angle shifts. The inaccuracy is largest if the realized measuring inaccuracies have opposite signs for the angle shifts of s- and p-polarization. This can also be seen in fig. 2 where the intersection point shifts to the same direction if the curve for p-polarization is shifted to the right or the curve for s-polarization to the left.

The measuring accuracy is highest if the resonance angles of the bare and covered $\mathrm{SiO}_{2}$ are measured in one run and no repositioning of the layerstructure in the measuring instrument has to take place. This is possible if layer 4 only partly covers layer 3 .

Now that it is clear that the overall accuracy of the method is determined by the accuracy of the angle shifts due to layer 4 an expression can be derived to calculate the accuracy of the obtained thickness and dielectric constant for an arbitrary layer 4 (see appendix):

$\left|\frac{\Delta d_{4}}{d_{4}}\right|=\frac{1}{\epsilon_{4}-\epsilon_{5}}\left(\epsilon_{5}\left|\frac{\Delta\left(\Delta \theta_{\mathrm{s}}\right)}{\Delta \theta_{\mathrm{s}}}\right|+\epsilon_{4 \mathrm{e}}\left|\frac{\Delta\left(\Delta \theta_{\mathrm{p}}\right)}{\Delta \theta_{\mathrm{p}}}\right|\right)$,

$\left|\frac{\Delta \epsilon_{4}}{\epsilon_{4}}\right|=\left|\frac{\Delta\left(\Delta \theta_{\mathrm{s}}\right)}{\Delta \theta_{\mathrm{s}}}\right|+\left|\frac{\Delta\left(\Delta \theta_{\mathrm{p}}\right)}{\Delta \theta_{\mathrm{p}}}\right|$.

For the results of table 1 with $\Delta(\Delta \theta)=0.02^{\circ}$, eq. (2) gives $\Delta d / d=19 \%$ resp. $\Delta \epsilon / \epsilon=12 \%$, which are values close to the simulated values $23 \%$ and $11 \%$, respectively, presented in table 1 . This means that the approximate equations (2) may be used for accuracy analysis.

From eqs. (2) it is clear that for dielectric constants $\epsilon_{4}$ close to $\epsilon_{5}$ the thickness cannot be deter-

Table 1

Influence of angle changes on the characterization of layer 4 .

\begin{tabular}{|c|c|c|c|c|c|c|}
\hline \multirow{2}{*}{$\begin{array}{l}\text { Structure } 1-2-5 \\
\mathrm{p}\end{array}$} & \multicolumn{2}{|c|}{ Structure 1-2-3-5 } & \multicolumn{2}{|c|}{ Structure 1-2-3-4-5 } & \multicolumn{2}{|c|}{ Deviation from original value } \\
\hline & $\mathbf{p}$ & s & $\mathbf{p}$ & s & $d_{4}$ & $\epsilon_{4}$ \\
\hline $\begin{array}{l}\theta_{2 \mathrm{p}}+0.04^{\circ} \\
\theta_{2 \mathrm{p}}+0.04^{\circ} \\
\theta_{2 \mathrm{p}}+0.04^{\circ} \\
\theta_{2 \mathrm{p}}+0.04^{\circ}\end{array}$ & $\begin{array}{l}\theta_{3 \mathrm{p}} \\
\theta_{3 \mathrm{p}}+0.04^{\circ} \\
\theta_{3 \mathrm{p}} \\
\theta_{3 \mathrm{p}}\end{array}$ & $\begin{array}{l}\theta_{3 \mathrm{~s}} \\
\theta_{3 \mathrm{~s}}+0.04^{\circ} \\
\theta_{3 \mathrm{~s}} \\
\theta_{3 \mathrm{~s}}\end{array}$ & $\begin{array}{l}\theta_{4 \mathrm{p}} \\
\theta_{4 \mathrm{p}}+0.04^{\circ} \\
\theta_{4 \mathrm{p}}+0.02^{\circ} \\
\theta_{4 \mathrm{p}}+0.02^{\circ}\end{array}$ & $\begin{array}{l}\theta_{4 \mathrm{~s}} \\
\theta_{48}+0.04^{\circ} \\
\theta_{4 \mathrm{~s}}+0.02^{\circ} \\
\theta_{48}-0.02^{\circ}\end{array}$ & $\begin{array}{l}0.4 \% \\
3 \% \\
9 \% \\
23 \%\end{array}$ & $\begin{array}{l}0.2 \% \\
2 \% \\
2 \% \\
11 \%\end{array}$ \\
\hline
\end{tabular}


mined accurately. For biological samples dielectric constants of 2-2.3 are expected which makes this method inaccurate if physiological buffers $(\epsilon=1.8)$ are used for medium 5 instead of air.

\section{Results}

Two gratings of different thickness were characterized with the method described here; the results are presented in table 2 .

The values found for the dielectric constant lie close to the value given by the manufacturer for a $1.5 \mu \mathrm{m}$ photoresist layer. The thickness of the first layer compares well with the value found with the surface profiler, the second grating could not be measured with the surface profiler, but was spincasted from a three times less concentrated photoresist solution than used for the other grating, which normally leads to about four times less thick layers $\left(d_{4} \approx 3.5 \mathrm{~nm}\right)$.

\section{Conclusions}

We have presented a method for characterization of a thin transparent dielectric layer by its thickness and dielectric constant. The results of the characterization of photoresist test layers compare very well with the dielectric constant given by the manufacturer and the thicknesses which were expected. With this method images of a thin layer can be made, so that it can be characterized spatially resolved.

The accuracy of the method is highest for samples measured in air. For very thin layers of a few $\mathrm{nm}$ it is necessary to have a more accurate method of measuring the shift in resonance angle. This can be done by integrating the turntable in a microscope setup (accuracy $0.01^{\circ}$ ) or integrating a vibrating mirror in a microscope setup (accuracy $0.002^{\circ}$ [11]).

The application of the method will become easier if flat $\mathrm{SiO}_{2}$ layers can be prepared, which can be characterized on the turntable setup. In this way standard substrates will become available for characterizing thin biological layers.

As $\mathrm{SiO}_{2}$ is a stable material the substrates can be cleaned and used for more than one experiment.

The SPR technique in which changes of the dielectric constant of the bulk medium are used to obtain $\epsilon$ and $d$ [6], can also be combined with microscopy. The difference is that in this case the SPR has to be performed in liquid bulk media where the here described method has the best performance for samples in air.

\section{Appendix}

In order to find an expression for the accuracy of the characterization of layer 4 the equations of the intersecting lines in the $\epsilon_{4}-d_{4}$ plane are necessary. Thiefenthaler and Lukosz [2] show that for thin layers 4 the first order approximation of the lines are

s-polarization: $d_{4}\left(\epsilon_{4}-\epsilon_{5}\right)=$ constant, p-polarization:

$d_{4} \frac{\left(\epsilon_{4}-\epsilon_{5}\right)}{\epsilon_{4}}\left[\epsilon_{4}\left(\frac{1}{\epsilon_{5}}-\frac{1}{N^{2}}\right)+1\right]=$ constant,

$N=n_{1} \sin \theta_{\text {res }}=k_{x} \lambda / 2 \pi$.

In our case $\mathrm{SiO}_{2}$ layers of about $315 \mathrm{~nm}$ thick are used. For this layer the resonance angle for p-polarization lies close to the critical angle $\theta_{c}=$ $\arcsin \sqrt{\epsilon_{5} / \epsilon_{1}} ;$ this means that $N \approx \sqrt{\epsilon_{5}}$.

Table 2

Experimental results of photoresist gratings. The angles are angles with the normal of the prism interface.

\begin{tabular}{|c|c|c|c|c|c|c|c|c|}
\hline \multirow[t]{2}{*}{ Grating } & \multirow[t]{2}{*}{$\theta_{3 \mathrm{p}}$} & \multirow[t]{2}{*}{$\theta_{3 \mathbf{z}}$} & \multirow[t]{2}{*}{$\theta_{4 p}$} & \multirow[t]{2}{*}{$\theta_{43}$} & \multicolumn{2}{|c|}{ New method } & \multicolumn{2}{|c|}{ Control } \\
\hline & & & & & $d_{4}(\mathrm{~nm})$ & $\epsilon_{4}$ & $d_{a}^{a}$ & $\epsilon_{4}^{b}$ \\
\hline I & -2.46 & 20.03 & -0.79 & 22.02 & $14.2 \pm 0.4$ & $2.70 \pm 0.06$ & $14 \pm 1$ & $\approx 2.79$ \\
\hline II & 0.10 & 22.56 & 0.60 & 23.03 & $3.5 \pm 0.5$ & $2.78 \pm 0.28$ & - & $\approx 2.79$ \\
\hline
\end{tabular}

a) Thickness measured with a surface profiler. ") Dielectric constant given by Shipley for $1.5 \mu \mathrm{m}$ thick photoresist layers. 
To find the accuracy in obtaining the intersection point equations ( 3 ) are linearized around the intersection point $\left(\epsilon_{4 \mathrm{e}}, d_{4 \mathrm{e}}\right)$ :

$\epsilon_{4 \mathrm{~s}}-\epsilon_{4 \mathrm{e}}=\epsilon_{4 \mathrm{~s}}^{\prime}\left(d_{4}-d_{4 \mathrm{e}}\right)$,

$\epsilon_{4 \mathrm{p}}-\epsilon_{4 \mathrm{e}}=\epsilon_{4 \mathrm{p}}^{\prime}\left(d_{4}-d_{4 \mathrm{e}}\right)$,

with

$\epsilon_{4 \mathrm{~s}}^{\prime}=-\left(\epsilon_{4 \mathrm{e}}-\epsilon_{5}\right) / d_{4 \mathrm{e}}$,

$\epsilon_{4 \mathrm{p}}^{\prime}=-\left(\epsilon_{4 \mathrm{e}}-\epsilon_{5}\right) \epsilon_{4 \mathrm{e}} / d_{4 \mathrm{e}} \epsilon_{5}$.

Equations (3) show that for a given $\epsilon_{4}, d_{4}$ depends linearly on the angle shift for both polarizations as for small angle shifts the constants are linearly dependent on the angle shifts. So

$\left|\frac{\Delta d_{4}}{d_{4}}\right|=\left|\frac{\Delta\left(\Delta \theta_{\text {res }}\right)}{\Delta \theta_{\text {res }}}\right|$.

The largest inaccuracies are obtained if the lines for s- and p-polarization shift in opposite directions (see fig. 2 and table 1 ).

The shifted curves

$\epsilon_{4 \mathrm{~s}}-\epsilon_{4 \mathrm{e}}=\epsilon_{4 \mathrm{~s}}^{\prime}\left(d_{4}-d_{4 \mathrm{e}}+d_{\mathrm{s}}^{*}\right)$,

$\epsilon_{4 \mathrm{p}}-\epsilon_{4 \mathrm{e}}=\epsilon_{4 \mathrm{p}}^{\prime}\left(d_{4}-d_{4 \mathrm{e}}-d_{\mathrm{p}}^{*}\right)$,

give the new intersection point:

$d_{4}=d_{4 \mathrm{e}}+\frac{\epsilon_{4 \mathrm{~s}}^{\prime} d_{\mathrm{s}}^{*}+\epsilon_{4 \mathrm{p}}^{\prime} d_{\mathrm{p}}^{*}}{\epsilon_{4 \mathrm{p}}^{\prime}-\epsilon_{4 \mathrm{~s}}^{\prime}}$ $\epsilon_{4}=\epsilon_{4 e}-\left(\epsilon_{4 e} / d_{4 e}\right)\left(d_{s}^{*}+d_{p}^{*}\right)$,

Combination of eqs. (4), (5) and (7) leads to

$\left|\frac{\Delta d_{4}}{d_{4}}\right|=\frac{1}{\epsilon_{4}-\epsilon_{5}}\left(\epsilon_{5}\left|\frac{\Delta\left(\Delta \theta_{\mathrm{s}}\right)}{\Delta \theta_{\mathrm{s}}}\right|+\epsilon_{4 \mathrm{e}}\left|\frac{\Delta\left(\Delta \theta_{\mathrm{p}}\right)}{\Delta \theta_{\mathrm{p}}}\right|\right)$,

$\left|\frac{\Delta \epsilon_{4}}{\epsilon_{4}}\right|=\left|\frac{\Delta\left(\Delta \theta_{s}\right)}{\Delta \theta_{s}}\right|+\left|\frac{\Delta\left(\Delta \theta_{p}\right)}{\Delta \theta_{p}}\right|$.

\section{References}

[1] H. Arwin and D.E. Aspnes, Thin Sol. Films 113 (1984) 101.

[2] K. Thiefenthaler and W. Lukosz, J. Opt. Soc. Am. B 6 (1989) 209.

[3] H.E. de Bruijn, B.S.F. Altenburg, R.P.H. Kooyman and J. Greve, Optics Comm. 82 (1991) 425.

[4] B. Rothenhäusler and W. Knoll, Nature 332 (1988) 615.

[5] A. Otto and W. Sohler, Optics Comm. 3 (1971) 254.

[6] H.E. de Bruijn, R.P.H. Kooyman and J. Greve, Appl. Optics 29 (1990) 1974.

[7] H.E. de Bruijn, A.T.M. Lenferink, R.P.H. Kooyman and J. Greve, Optics Comm. 86 (1991) 444.

[8] H.E. de Bruijn, R.P.H. Kooyman and J. Greve, in preparation.

[9] H.E. de Bruijn, R.P.H. Kooyman and J. Greve, Appl. Optics 31 (1992) 440.

[10] B. Rothenhäusler and W. Knoll, J. Opt. Soc. Am. B 5 (1988) 1401.

[11] R.P.H. Kooyman, A.T.M. Lenferink, R.G. Eenink and J. Greve, Ann. Chem. 63 (1990) 83. 